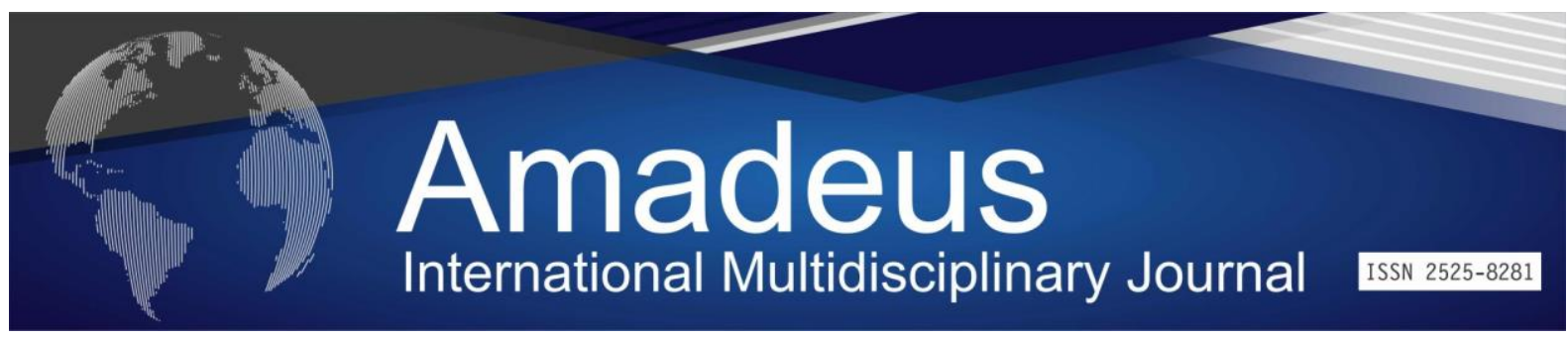

DOI: 10.14295/aimj.v4i7.94

\title{
Cutaneous Blastomycosis Simulating Ganglionic Tuberculosis: A Case Report
}

Luanna Angélica Pesqueira e Silva Modesto Leite Rolim Neto ${ }^{2}$ Pablo Pita

\begin{abstract}
Blastomycosis is a chronic or subacute deep mycosis caused by a dimorphic fungus called Blastomyces dermatitidis, which usually produces pulmonary disease and, to a lesser extent, extrapulmonary forms such as cutaneous, osteoarticular, genitourinary, among others. Case Report: The authors report a case of primary cutaneous blastomycosis in Brazil, with characteristics that mimic ganglion tuberculosis. The diagnosis was confirmed by biopsy and histopathology. Conclusion: Cutaneous blastomycosis is clinically similar to ganglion tuberculosis, thus being an important differential diagnosis. Thus, knowing the characteristics and particularities of the disease, as well as its epidemiology, are important factors in the diagnosis of the disease.
\end{abstract}

Keywords: Cutaneous blastomycosis, Ganglionic tuberculosis, Verrucous lesions.

\footnotetext{
${ }^{1}$ Graduação em Medicina pela Faculdade de Medicina Estácio Juazeiro do Norte, Brasil;

${ }^{2}$ Professor Livre Docente pela Faculdade de Saúde Pública da Universidade de São Paulo. Pós-Doutor em Saúde Pública pela Universidade de São Paulo - USP. Pós-Doutor em Lingüística pela Universidade Federal de Pernambuco - UFPE . Doutor em Ciências da Saúde pela Universidade Federal do Rio Grande do Norte - UFRN. Professor do Curso de Medicina da Universidade Federal do Cariri - UFCA. Professor do Curso de Medicina da Estácio/Faculdade de Medicina de Juazeiro do Norte - FMJ. Professor do Curso de Direito da Faculdade de Juazeiro do Norte - FJN. Autor correspondente: modestorolim@yahoo.com.br;

${ }^{3}$ Médico Infectologista. Mestrando em Ciências da Saúde pela Faculdade de Medicina do ABC - Santo André - SP. Pós-graduação pela Universidade de Pernambuco em Infectologia por meio de Residência Médica. É Coordenador do Programa de Residência Médica e Internado de Medicina Interna da Faculdade Estácio de Medicina, Juazeiro do Norte - CE.
} 


\section{Blastomicose Cutânea Simulando Tuberculose Ganglionar: Relato de Caso}

\begin{abstract}
Resumo: A blastomicose é uma micose profunda crônica ou subaguda causada por um fungo dimórfico chamado Blastomyces dermatitidis, que geralmente produz doença pulmonar e, em menor grau, formas extrapulmonares como a cutânea, osteoarticular, genitourinário, entre outros. Relato de Caso: Os autores relatam um caso de blastomicose cutânea primária no Brasil, com características que mimetizam tuberculose ganglionar. $\mathrm{O}$ diagnóstico foi confirmado através de realização de biópsia e histopatológico. Conclusão: A blastomicose cutânea se assemelha clinicamente à tuberculose ganglionar, sendo assim, um importante diagnóstico diferencial. Dessa forma, conhecer as características e particularidades da doença, assim como sua epidemiologia, são fatores importantes no diagnóstico da patologia.
\end{abstract}

Palavras-chave: Blastomicose cutânea, Tuberculose Ganglionar, Lesões verrucosas.

\section{Introdução}

Blastomyces dermatitidis é um fungo termicamente dimórfico que cresce como mofo no meio ambiente e como levedura nos tecidos. As áreas de alta endemicidade são encontradas na América do Norte, principalmente, os vales dos rios Mississipi e Ohio, os estados do meio oeste e as províncias canadenses que fazem fronteira com os Grandes Lagos e as áreas de Nova York e Canadá que são adjacentes à via marítima do rio St. Lawrence ${ }^{1}$. O fungo $B$. dermatitidis produz uma doença chamada blastomicose que possui como principal forma de aquisição, a inalação de conídios do solo produzidos na fase de mofo, sendo portanto, principalmente uma infecção pulmonar ${ }^{2,3}$. Classicamente, isso se manifesta como doença pulmonar primária com sintomas como: febre, tosse e fadiga, mas pode ser assintomático ${ }^{1,4}$.

Após a infecção do trato respiratório, quando as defesas alveolares não conseguem conter o microorganismo, o $B$. dermatitidis se dissemina por via hematogênica em locais extrapulmonares, em 20 a 50\% dos casos, na maioria das vezes na pele, ossos, articulações e sistema nervoso central ${ }^{1,4-7}$. As lesões cutâneas podem se desenvolver por meio da inoculação primária com o fungo ou disseminação da doença 
pulmonar primária ${ }^{1,4}$. A blastomicose cutânea apresenta-se tipicamente com formas verrucosas ou ulcerativas. As lesões verrucosas começam como pápulas que se desenvolvem em crosta e placas verrugosas de formato irregular e zona central clara. As lesões ulcerativas geralmente começam como pápulas eritematosas que ulceram em um padrão assimétrico e drenagem serossanguínea crônica ${ }^{1,5}$.

A ocorrência rara da blastomicose cutânea no Brasil, assim como as características peculiares da patologia, que simula uma tuberculose ganglionar, demonstram a necessidade de estudar e relatar o caso.

\section{Relato de Caso}

Paciente masculino, 47 anos, relata que em novembro de 2015 iniciou de forma súbita, o aparecimento de lesões nodulares em região cervical, enquanto trabalhava como tratorista numa fazenda de soja na cidade de Paragominas - Pará. Ao exame físico a lesão possuía aspecto endurecido, formato nodular, com bordas e centros elevados, caráter progressivo, com eritema de permeio. (Figura 1) O paciente não referiu prurido, mas sim, intenso processo inflamatório, calor e dor local, de leve intensidade, associado à drenagem de secreção purulenta com sangue, que evoluía para lesão não cicatrizante por toda extensão anterior cervical. (Figura 2).

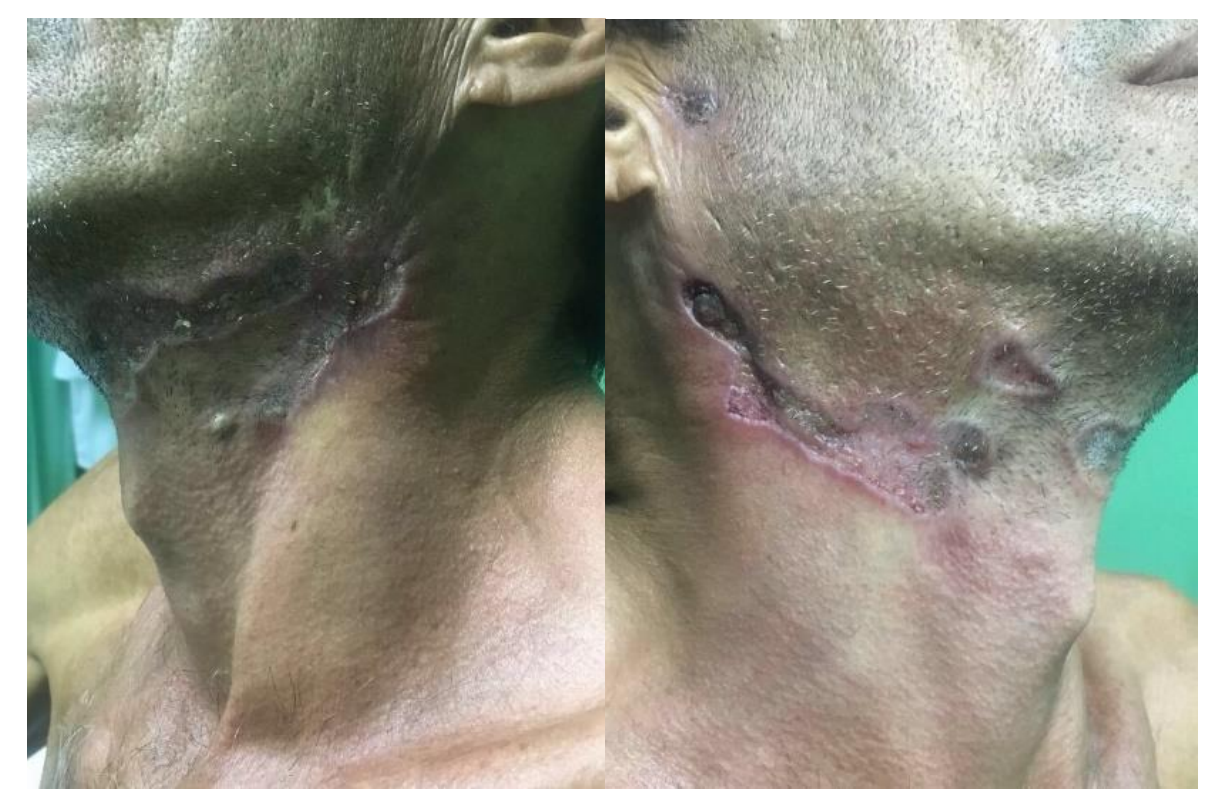

Figura 1. Vista lateral da lesão cervical 


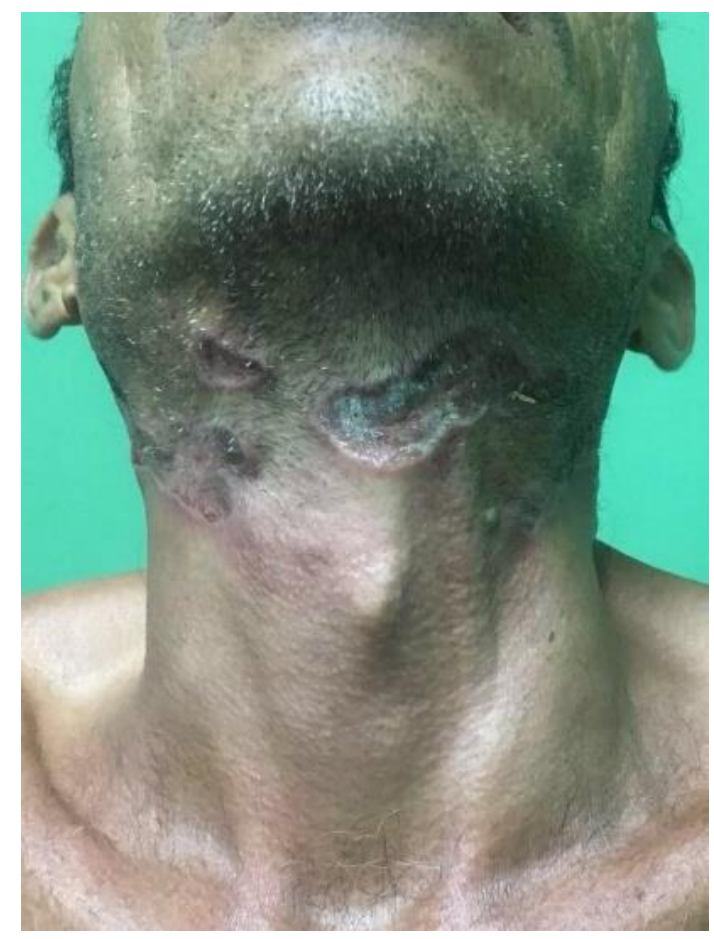

Figura 2. Vista anterior da lesão cervical

Associado a essa queixa apresentava episódios de febre intermitente, não aferida, e que cessava com o uso de analgésicos comuns, além de dores articulares. Paciente referiu que durante esse tempo fez uso de antibióticos e anti-inflamatórios, sem melhora do quadro. Relatou ainda episódios de tosse seca, às vezes produtiva com secreção branca e emagrecimento em torno de $20 \mathrm{~kg}$ em um ano. Em janeiro de 2016, mesmo após realizar duas baciloscopias com amostra de escarro negativas, iniciou tratamento empírico para tuberculose (TB), por seis meses. Não concluiu o tratamento, pois referiu piora do quadro clínico após o início da medicação, apresentando astenia, intensificação das dores articulares e febre. Afirmou ainda que os nódulos em região cervical apresentaram piora clínica. Dessa maneira por conta própria suspendeu o tratamento para tuberculose em junho de 2016.

Realizou exames complementares como, exame radiológico de tórax dia 22/06/2016 que evidenciou infiltrado pneumônico reticular basal bilateral, e outro, realizado dia 09/08/2016 com espessamento das paredes brônquicas, alteração da transparência pulmonar, estrias e micronódulos nos ápices pulmonares. Em outubro de 2016 foi encaminhado para atendimento especializado na cidade de Brejo Santo - CE, com as lesões em região cervical ainda em atividade, sendo retratado para TB ganglionar com comprometimento pulmonar. Ao exame possuía linfonodomegalia 
cervical com drenagem e fístula. Então, em abril de 2017, sem melhora, foi solicitada uma biópsia de pele cervical evidenciando ao histopatológico - extensões de pele epidérmica pseudoepiteliomatosa com abscessos intraepidérmicos, com reação crônica inflamatória granulomatosa linfoplasmocítica com histiócitos e eosinófilos de permeio. Ocasionalmente células gigantes multinucleadas, não raro com levedura em seu interior lembrando, Blastomyces dermatitidis, doença de Gilchrist ou Blastomicose.

Foi então iniciado o tratamento com o antifúngico, Itraconazol 100mg, via oral, 2 comprimidos ao dia por no mínimo 12 meses. Após do início do tratamento o paciente retorna ao serviço evidenciando melhora da lesão cervical, cicatrizada sem recidiva das lesões e sem outras queixas. (Figura 3)

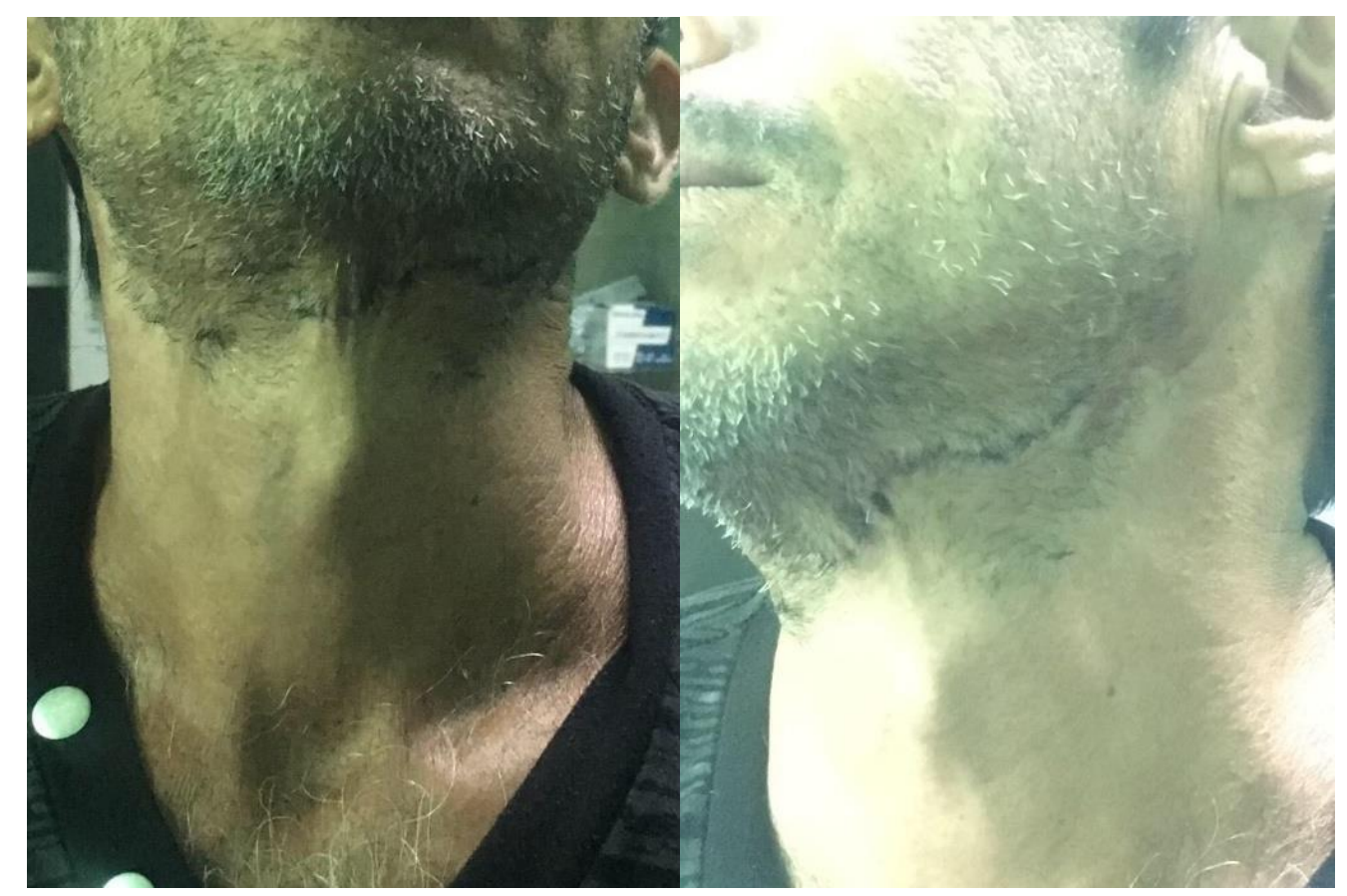

Figura 3. Lesão em fase final de tratamento, evidenciando regressão da área ulcerada.

\section{Discussão}

Blastomyces dermatitidis pode causar infecção endêmica em indivíduos expostos. Embora o sítio mais comum de infecção seja pulmonar, pode ocorrer infecção extrapulmonar, incluindo pele, ossos e sistema nervoso central ${ }^{8}$. 
Dentre os casos relatados endemicamente, o paciente típico com blastomicose é um homem de meia idade que participa de atividades recreativas ou ao ar livre que sejam próximas de corpos de água doce. Não está totalmente esclarecido se essa associação representa um verdadeiro vínculo causal com relação ao risco de transmissão ou se é simplesmente porque muitas atividades ao ar livre populares envolvem água doce ${ }^{1}$.

Um estudo realizado na Universidade de Arkansas demonstrou uma prevalência de $58 \%$ dos casos de blastomicose em homens, dado semelhante aos de estudos realizados em Manitoba e Ontário que também demonstraram a maior prevalência da doença em homens do que em mulheres ${ }^{9,10}$.

Uma das hipóteses para a predominância do sexo masculino na blastomicose, seria o fato de que os homens são mais propensos a participar de atividades associadas à exposição por B. dermatitidis, como caça, pesca e agricultura ${ }^{1}$.

A blastomicose cutânea primária (BCP) é uma entidade particularmente rara, que pode ocorrer através de mordidas de animais infectados ou inoculação direta em ambientes laboratoriais ${ }^{11,12}$.

A inoculação acidental como resultado de lesões diretas no contexto da atividade ao ar livre envolvendo solo também pode ocorrer. Existem quatro critérios que devem ser cumpridos ao fazer um diagnóstico de inoculação primária por blastomicose: (1) presença de lesões cutâneas, muitas vezes com linfadenopatia focal; (2) história de inoculação com material conhecido por conter o organismo fúngico; (3) ausência de envolvimento sistêmico; e (4) recuperação de organismos da lesão ${ }^{12}$.

A histopatologia e a morfologia lesional não podem distinguir a inoculação primária da doença cutânea disseminada, no entanto, a inoculação direta é mais provável que resulte clinicamente em linfangite local ${ }^{4,12}$.

Em relação ao caso relatado neste estudo, o paciente apresentou lesões cutâneas, de início súbito, sem envolvimento pulmonar inicial, fato que justificou a realização de biópsia e suspeita de blastomicose cutânea primária.

A BCP geralmente começa com a inoculação de fungos na pele devido a um traumatismo. Após um período de 2 a 3 semanas, desenvolve-se um cancro acompanhado de linfagite e linfadenite. A principal manifestação clínica é a lesão verrugosa, mas pode ser ainda nodular, papular e apenas alguns pacientes apresentam lesões pustulares ${ }^{13}$. As lesões cutâneas são frequentemente subcutâneas, localizadas no rosto, antebraços, mãos e pernas dos pacientes e podem desenvolver ulceração, como 
demonstrado no caso relatado. Quando localizada na cabeça, as lesões podem atingir mucosas como nariz, boca ou laringe.

As lesões verrugosas da faringe podem ser confundidas com câncer de células escamosas. Uma úlcera cutânea é formada a partir de um abscesso subcutâneo que drena através da pele. Estas úlceras apresentam bordas amassadas com ou sem base exsudativa ${ }^{1}$.

Apesar da grande quantidade de infecções por fungos ocorrerem em pacientes imunocomprometidos, a blastomicose apresenta-se em indivíduos que possuem imunidade normal. É o que demonstra também uma revisão de 20 anos, em que dos 123 pacientes estudados, apenas 31 (25\%) foram relatados com imunossupressão ${ }^{4,8,14,15}$.

Um diagnóstico diferencial da blastomicose é a tuberculose ganglionar. Esta patologia, diferentemente da blastomicose, ocorre em pacientes imunodeficientes relacionados à AIDS. A tuberculose ganglionar é a segunda forma mais comum de tuberculose, atingindo frequentemente os gânglios da cadeia cervical anterior, com leve predomínio à direita. Os gânglios são inicialmente móveis e indolores, possuem um crescimento lento e tendem a coalescer, formando uma massa que se adere aos planos superficial e profundo.

O quadro clínico geralmente se apresenta de forma insidiosa, com febre, emagrecimento e com aumento progressivo dos linfonodos que se transformam em massas linfonodais. Se não houver tratamento, a massa ganglionar fistuliza e drena material seroso ou purulento ${ }^{16,17}$.

O paciente do caso em questão foi tratado com Itraconazol, corroborando as evidências atuais de que esta é a droga de escolha para casos cutâneos primários de blastomicose, com uma dosagem de 100-300 mg/dia, obtendo uma boa resposta em três meses $^{13}$.

\section{Conclusão}

A blastomicose cutânea primária é uma entidade rara no Brasil. Apresenta sintomas e sinais clínicos que mimetizam a tuberculose ganglionar.

Dessa forma, a suspeita diagnóstica a partir de dados epidemiológicos e característica da lesão, deve ser realizada de forma precoce para evitar um período 
prolongado sem o tratamento correto para o paciente, agravando assim, o quadro clínico do mesmo.

\section{Referências}

1. Saccente M, Woods GL. Clinical and laboratory update on blastomycosis. Clin Microbiol Rev. 2010 Apr; 23 (2): 367-81.

2. Kauffman CA. Treatment of the Midwestern Endemic Mycoses, Blastomycosis and Histoplasmosis. Curr Fungal Infect Rep. 2017; 1-8.

3. Smith JA, Gauthier G. New developments in blastomycosis. Sem Respir Crit Care Med. 2015 Out; 36 (5): 715-28.

4. Smith RJ, Boos MD, Burnham JM, McKay EM, Kim J, Jen M. Atypical Cutaneous Blastomycosis in a Child With Juvenile Idiopathic Arthritis on Infliximab. Pediatrics. 2015 Nov; 136 (5): e1386-9.

5. Mason AR, Cortes GY, Cook J, Maize JC, Thiers BH. Cutaneous blastomycosis: a diagnostic challenge. Int J Dermatol. 2008;47(8):824 830.

6. Siemieniuk RAC, MacFadden DR, Matukas LM. Disseminated blastomycosis with cutaneous involvement in a Canadian railroad worker. The Lancet. 2015; 385 (9971): 883.

7. Stephens J, Huddleston H, Reeck M. Cutaneous blastomycosis in the absence of active pulmonary disease. J Am Acad Dermatol. 2007; 56 (2): supl. 2, AB130.

8. Zhu D, Chen H, Yu L. Probable pulmonary Blastomyomycocis in an immunocompetent person. Int J Infect Dis. 2017; 59: 86-89.

9. Crampton TL, Light RB, Berg GM, Meyers MP, Schroeder GC, Hershfield ES, et al. Epidemiology and clinical spectrum of blastomycosis diagnosed at Manitoba hospitals. Clin. Infect. Dis. 2002; 34 (10): 1310-1316.

10. Morris SK, Brophy J, Richardson SE, Summerbell R, Parkin PC, Jamieson F, et al. Blastomycosis in Ontario, 1994-2003. Emerg. Infect. Dis. 2006; 12 (2): 274-279.

11. Ferchichi L, Mekni A, Bellil K, Haouet S, Zeddini A, Bellil S, et al. La blastomycose cutanée: trois cas. Med Mal Infect. 2006; 36 (5): 285-287.

12. Gray NA, Baddour LM. Cutaneous inoculation blastomycosis. Clin Infect Dis. 2002; 34 (10): E44-49. 
13. Bonifaz A, Morales D, Morales N, Mercadillo P, González GM, HernándezHernández F, et al. Cutaneous blastomycosis. An imported case with good response to itraconazole. Rev Iberoam Micol. 2016; 33 (1): 51-4.

14. Chapman SW, Dismukes WE, Proia LA, Bradsher RW, Pappas PG, Threlkeld MG, et al. Clinical practice guidelines for the management of blastomycosis: 2008 update by the Infectious Diseases Society of America. Clin Infect Dis. 2008; 46 (12): $1801-12$.

15. Lemos LB, Baliga M, Guo M. Blastomycosis: the great pretender can also be na opportunist. Initial clinical diagnosis and underlying diseases in 123 patients. Ann Diagn Pathol. 2002; 6 (3): 194-203.

16. Campos HS. Etiopatologia da tuberculose e formas clínicas. Pulmão. 2006; 15 (1): 29-35.

17. Capone D, Mogami R, Lopes AJ, Tessarollo B, Cunha DL, Capone RB, et al. Tuberculose extrapulmonar. Revista Hospital Universitário Pedro Ernesto. 2006;5(2):54-67.

\section{How to cite this article (APA format):}

Silva, Luanna Angélica Pesqueira e; Rolim Neto, Modesto Leite; Pita, Pablo. (2019). Blastomicose Cutânea Simulando Tuberculose Ganglionar: Relato de Caso. Am. In. Mult. J., October a December. (7) 4, 245-253.

Received: 12/02/2019

Accepted: 15/12/2019 